\title{
TENDINOPATIA DO COMPARTIMENTO ANTERIOR DO TORNOZELO
}

\author{
TENDINOPATHY OF THE ANTERIOR COMPARTMENT OF THE ANKLE
}

Antonio Egydio de Carvalho Junior ${ }^{1}$, Cíntia Kelly Bittar ${ }^{2}$, Osny Salomão ${ }^{3}$, João Batista Miranda ${ }^{4}$, André Ninomiya ${ }^{5}$, Daniel Bento Silva ${ }^{6}$

\section{RESUMO}

Objetivo: Análise retrospectiva da etiopatogenia, diagnóstico e opções de tratamento nos casos de tendinopatias do compartimento anterior do tornozelo (TCAT). Método: No período de setembro de 1998 a fevereiro de 2009, 13 pacientes foram operados por tendinopatia do compartimento anterior do tornozelo. A casuística constou de 10 pacientes do sexo masculino e três do feminino. O lado direito foi acometido em 12 pés e um do esquerdo. A média de idade foi de 35 anos (15-67). A etiologia foi traumática em oito pacientes e em cinco, degenerativa (atraumática). O tempo médio do diagnóstico ao tratamento foi de 19 meses (1-60) e o seguimento foi de 34 meses (4-127). O diagnóstico foi feito através da história e exame clínico. A ressonância magnética foi realizada em nove pacientes para estadiamento e planejamento. O tratamento cirúrgico foi personalizado para cada caso (sinovectomia, ressecção de ventre muscular, solidarização com o tendão adjacente e enxerto livre de tendão semitendíneo). Para a avaliação dos resultados foram utilizadas as escalas: 1) graduação subjetiva de satisfação, 2) AOFAS e 3) Maryland. Resultado: Em relação à escala de graduação subjetiva de satisfação, 12 pacientes satisfeitos e um paciente insatisfeito. A média da escala AOFAS foi de 80 pontos, a média da escala Maryland foi de 86 pontos. Conclusão: O tratamento cirúrgico é eficaz para recuperação funcional. As técnicas cirúrgicas devem ser personalizadas. A opção do enxerto livre de tendão semitendíneo é eficiente nas falhas maiores que cinco centímetros.

Descritores - Síndrome do compartimento anterior; Tendinopatia; Cirurgia ortopédica; Transferência de tendão

\section{ABSTRACT}

Objective: To carry out a retrospective analysis of the etiopathogeny, diagnosis and therapeutic options in cases of tendinopathies of the anterior compartment of the ankle. Method: 13 patients underwent surgery between September 1998 and February 2009; ten men and three women. The right side was involved in twelve patients and the left in one. The averaging age was 35 years of age (15-67). The etiology was traumatic in eight patients and degenerative (non-traumatic) in five. The average time to diagnosis was 19 months $(1-60)$ and the average follow-up time was 34 months (4 -127). The diagnosis was made by anamnesis and clinical findings. Magnetic resonance imaging was performed in nine patients, for staging and planning. The surgical treatment was personalized in each case (debridement, resection of the muscle, grafting with the adjacent tendon, and free graft of the semitendinosus tendon). The following scales were used for the evaluation: 1) subjunctive satisfaction scale, 2) AOFAS and 3) Maryland. Results: In relation to the subjective satisfaction scale, 12 patients were satisfied and one was dissatisfied. The average AOFAS scale was 80 points, and the average Maryland scale was 86 points. Conclusion: Surgical treatment is effective for restoring function. The surgical techniques must also be personalized. The free graft of the semitendinosus tendon option is effective for gaps larger than five centimeters.

Keywords - Anterior compartment syndrome; Tendinopathy; Orthopaedic surgery; Tendon transfer

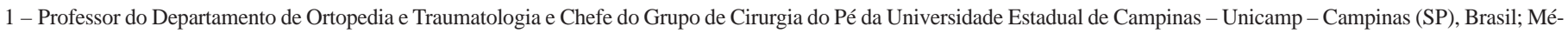
dico Assistente do Instituto de Ortopedia e Traumatologia do Hospital das Clínicas da Faculdade de Medicina da Universidade de São Paulo - USP - São Paulo (SP), Brasil.

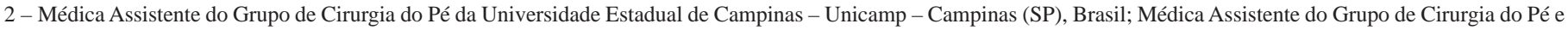
Tornozelo do Serviço de Ortopedia do Hospital e Maternidade Celso Pierrô da Pontifícia Universidade Católica de Campinas - PUC - Campinas - Campinas (SP), Brasil.

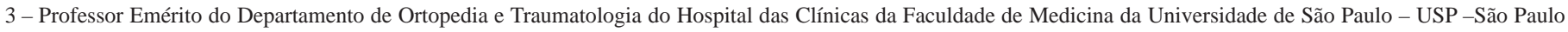
(SP), Brasil.

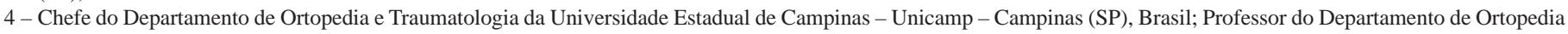
e Traumatologia e Chefe do Grupo de Cirurgia do Joelho da Universidade Estadual de Campinas - Unicamp - Campinas (SP), Brasil.

5 - Médico Estagiário do Grupo de Cirurgia do Pé da Universidade Estadual de Campinas - Unicamp - Campinas (SP), Brasil.

6 - Médico Estagiário do Grupo de Cirurgia do Pé da Universidade Estadual de Campinas - Unicamp - Campinas (SP), Brasil.

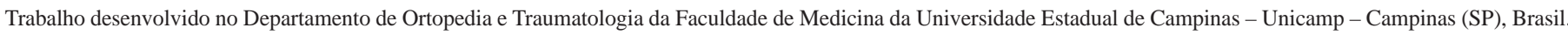
Correspondência: Rua Guarara, 261 - apto. 82 - Jd. Paulista - 01425-001 - São Paulo, SP - E-mail: footsport@uol.com.br 


\section{INTRODUÇÃO}

O compartimento anterior do tornozelo delimita um túnel cujo assoalho corresponde à face anterior da metáfise da tíbia e o teto, também inelástico, é formado pela expansão do ligamento retinacular anterior e por seus correspondentes feixes superior e inferior. Transitam por este segmento os tendões dos músculos tibial anterior (TA), extensor longo do hálux (ELH) e extensor longo dos dedos (ELD) nas suas respectivas bainhas sinoviais e o feixe vasculonervoso. $\mathrm{O}$ aumento das pressões dentro do túnel é resultante de tenossinovite, rotura, variação anatômica, expansão capsular, osteófito marginal anterior da tíbia, etc. O nervo fibular profundo pode ser comprimido e ocasionar dor e disfunção.

O diagnóstico da rotura tendínea se confirma através de dor e fraqueza da extensão do tornozelo e dedos ${ }^{(1)}$.

De maneira complementar, a ressonância magnética tem sido usada para o reconhecimento das lesões possíveis e seu estadiamento. Quando se trata de rotura, a imagem identifica o distanciamento entre os cotos e o grau intrínseco de estiramento tendíneo (esgarçamento), que são informações úteis para o planejamento terapêutico. Mesmo consideradas raras, as tendinopatias, neste compartimento, podem ser subestimadas ou apresentarem dificuldade para serem diagnosticadas quando pouco sintomáticas. O diagnóstico tardio deve ser diferenciado de outros achados, tais como cistos artrossinoviais, síndromes tumorais da parte anterior do tornozelo e compressões do nervo fibular profundo ${ }^{(2)}$.

As eventualidades terapêuticas ficam restritas aos sintomas e às incapacidades funcionais, não se levando em consideração idade, sexo, nível de atividade, comorbidades ou atraso no diagnóstico. As roturas completas devidamente estadiadas têm no tratamento cirúrgico o método de eleição na preferência dos autores ${ }^{(1,3,4)}$. Entretanto, Markarian et al ${ }^{(5)}$, Burman (1934) e Moskowitz (1971) apud Kashyap e Prince ${ }^{(2)}$ relatam bons resultados com tratamento conservador de tendinopatias e afirmam que o tratamento não cruento pode ser uma boa alternativa para os pacientes idosos de baixa demanda funcional.

A motivação deste estudo é descrever o arsenal de táticas cirúrgicas empregadas no tratamento das tendinopatias do compartimento anterior. As técnicas cirúrgicas utilizadas foram o desbridamento tendíneo, a ressecção do ventre muscular anômalo, a solidarização e, nas falhas maiores que cinco centímetros, optou-se pelo enxerto livre do tendão semitendíneo.

\section{OBJETIVO}

Analisar retrospectivamente a etiopatogenia, o diagnóstico e as opções de tratamento nos casos de tendinopatias do compartimento anterior do tornozelo.

\section{CASUÍSTICA E MÉTODOS}

No período de setembro de 1998 a fevereiro de 2009, 14 pacientes foram diagnosticados com tendinopatia do compartimento anterior do tornozelo. O tratamento cirúrgico foi indicado em 13 pacientes e houve um tratamento conservador devido a lesão parcial do tendão extensor dos dedos. Neste estudo, o critério de inclusão limitou-se aos pacientes operados.

A casuística constou de 10 pacientes do sexo masculino e três do feminino, sendo o lado direito acometido em 12 pés e um à esquerda. A média de idade foi de 35 anos (15-67).

A etiologia foi traumática em oito pacientes e o restante apresentava indícios de causa degenerativa (dois) e processo inflamatório (três), ambas consideradas atraumáticas.

O tempo médio do diagnóstico ao tratamento foi de 19 meses (1-60) e o seguimento foi de 34 meses (4-127).

O diagnóstico, nas roturas aguda ou crônica foi feito através da história e exame clínico. Nos casos crônicos evidenciou-se a tríade de sinais: 1) nodulação, correspondente a hipertrofia do coto proximal do tendão lesado; 2) perda da silhueta dos tendões sob o retináculo extensor; e 3) vicariância dos tendões íntegros ${ }^{(1)}$ (Figura 1).

A ressonância magnética foi realizada em nove pacientes para estadiamento da lesão e planejamento cirúrgico (Figura 2).

A distribuição da etiopatogenia nos cinco casos atraumáticos foi: 1) um caso de sinovite vilonodular e implan-
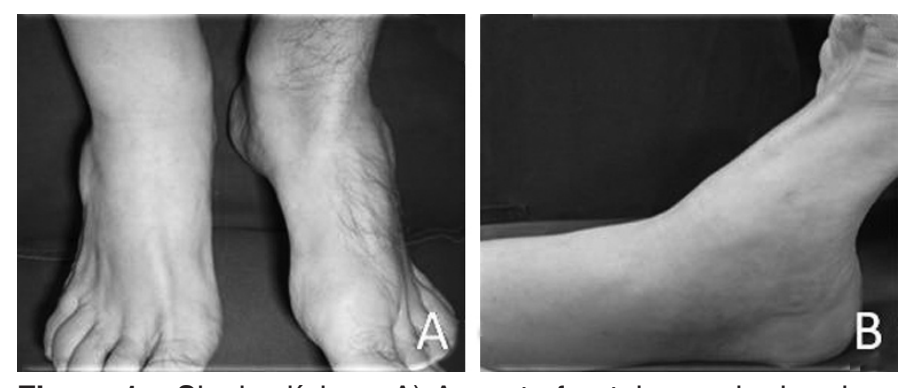

Figura 1 - Sinais clínicos: A) Aspecto frontal - perda do relevo do trajeto do tendão do tibial anterior. B) Aspecto lateral - vicariância dos tendões íntegros
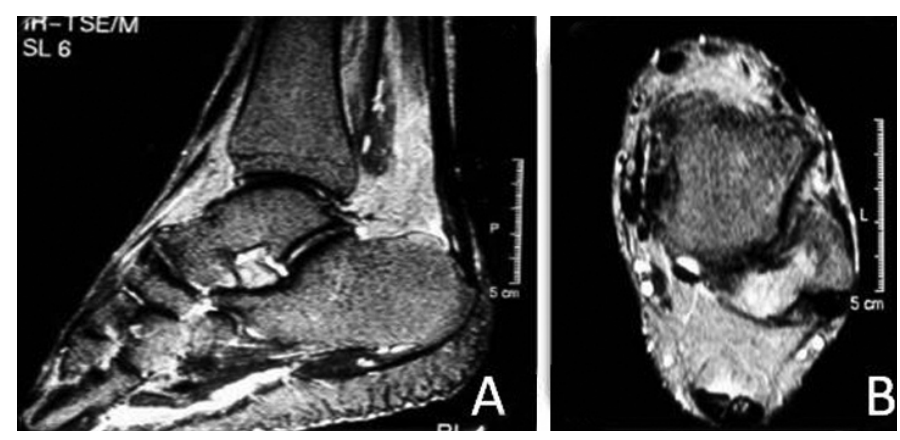

Figura 2 - Ressonância magnética: A) Projeção sagital: tenossinovite do tendão extensor longo dos dedos B) Projeção axial: processo inflamatório ao redor do tendão extensor dos dedos 
tação baixa do ventre muscular nos tendões tibial anterior e extensor longo dos dedos; 2) um caso de tenossinovite crônica do extensor longo dos dedos; 3) um caso de músculo acessório do tendão tibial anterior; e 4) dois casos de rotura degenerativa do tendão tibial anterior.

Nos pacientes sem rotura (2, 3 e 5), os fatores comuns foram gênero feminino, faixa etária semelhante (adulto jovem) e prática de atividades físicas de esforço. $\mathrm{O}$ exame físico desses pacientes mostrou que a dor era o sintoma presente no compartimento anterior, irradiada para a região dorsal do pé no trajeto correspondente ao nervo fibular profundo, evidenciada pelo sinal de Tinel. A incapacidade funcional era consequência do processo inflamatório e de variação anatômica que atuavam como fatores de compressão produzida pelo aumento do conteúdo em um continente inelástico.

Os casos atraumáticos (6 e 7) foram pacientes do sexo masculino, acima dos 60 anos, que apresentaram rotura espontânea do tendão tibial anterior durante a marcha. O exame clínico mostrou a tríade característica de alteração da silhueta, vicariância dos tendões adjacentes íntegros e nodulação. A etiopatogenia foi atribuída a um fator degenerativo inespecífico.

No grupo traumático havia referência de ferimento cortocontuso (FCC). A impotência funcional para a dorsiflexão do tornozelo ou dedos foi o sinal presente. No caso 13, havia perda de substância (pele total e segmentos dos tendões tibial anterior e extensor longo do hálux).

O comprometimento topográfico dos tendões acometidos mostrou:

a) Três roturas isoladas do tendão tibial anterior;

b) Duas secções isoladas e completas do tendão extensor longo dos dedos;

c) Duas roturas do tendão tibial anterior associadas ao tendão extensor longo dos dedos; e

d) Uma rotura do tendão tibial anterior completa associada à lesão parcial dos tendões extensor longo do hálux e dos dedos.

\section{TRATAMENTO}

No grupo atraumático, sem rotura, após o insucesso do tratamento conservador, a exploração cirúrgica foi indicada. Naquelas que apresentavam roturas de causa degenerativa, um paciente adiou voluntariamente a indicação cirúrgica inicial até sentir a sua necessidade e o outro aguardou o período de 30 dias de imobilização gessada.

O retardo da operação, nos casos traumáticos, ocorreu devido a diversos fatores como atraso do diagnóstico preciso, negligência do paciente e ao início tardio dos sintomas.
No paciente 13, a cirurgia foi retardada devido às condições de partes moles e foi necessário enxerto de tendões e retalho microvascular para cobertura cutânea, simultaneamente à reparação tendínea.

A tática cirúrgica empregada no caso 2 foi a ressecção do ventre do músculo tibial anterior e liberação do nervo fibular profundo. No caso 3 a cirurgia descompressiva constou da liberação do nervo fibular profundo, ressecção do ventre muscular e sinovectomia (ablação tumor intra-articular) (Figuras 3, 4 e 5).

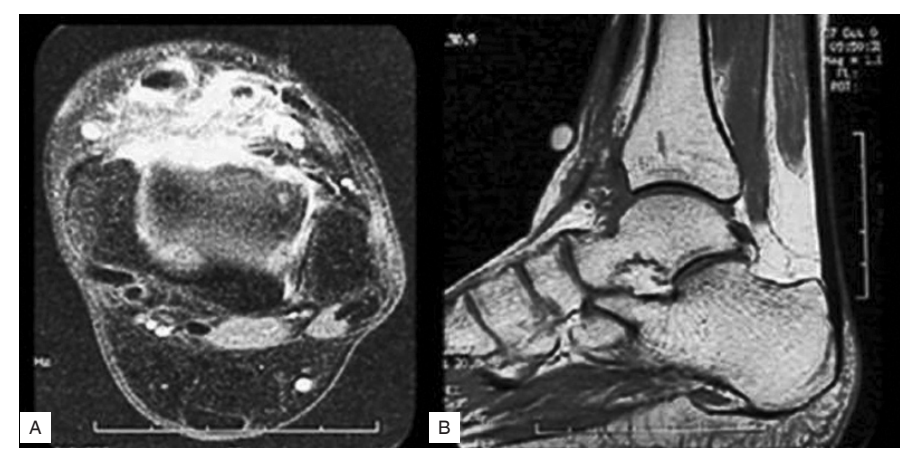

Figura 3 - Imagem de ressonância magnética: A) No plano axial; B) No sagital, mostrando ventre muscular baixo e tumor intra-articular

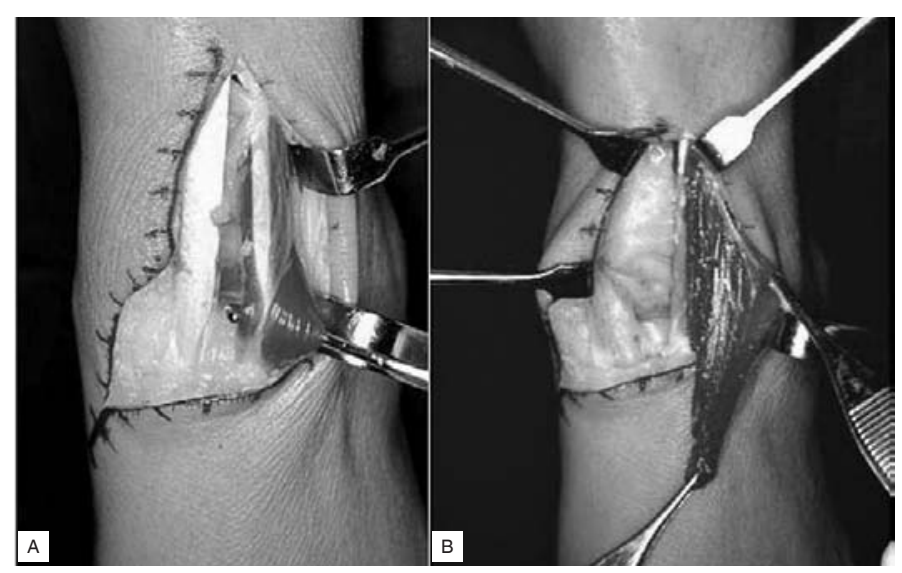

Figura 4 - A) Implantação baixa do ventre muscular; B) Ressecção do músculo anômalo

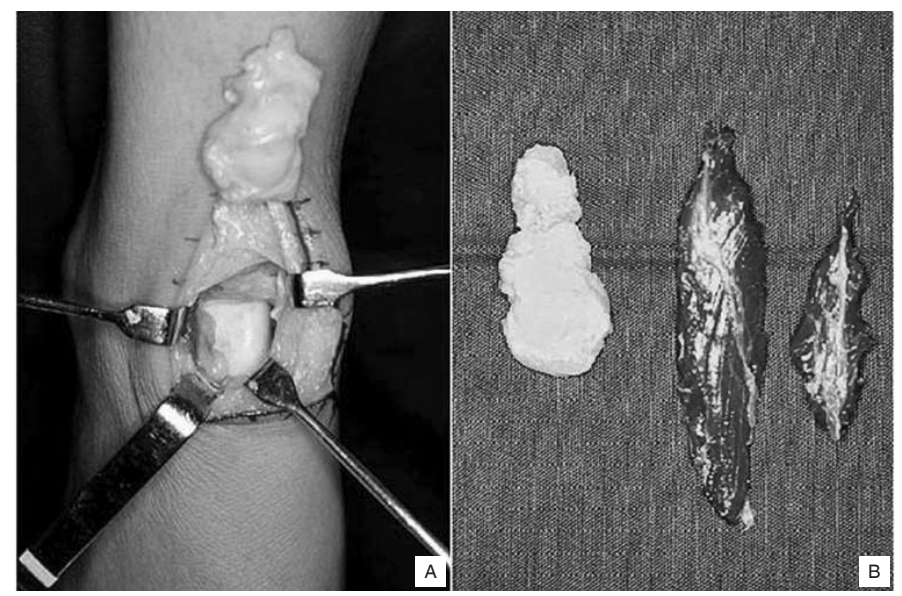

Figura 5 - A) Abertura da cápsula articular, ressecção de tumor vilonodular; B) peças retiradas 
No caso 5 foi feita ressecção da bainha tendínea e sinovectomia do extensor longo dos dedos.

Em todos os casos de rotura por ferimento cortante ou degenerativas, a reconstrução dos tendões foi realizada, pela solidarização com o tendão adjacente (casos 1, 4, 6, 8 e 9) ou por enxerto livre de semitendíneo (casos 7, 10, 11, 12 e 13). Nesses, a falha entre os cotos tendíneos, após o desbridamento, foi maior que cinco centímetros.

A técnica cirúrgica do enxerto livre do tendão semitendíneo consistiu em:

1. Identificação e retirada do tendão semitendíneo do joelho ipsilateral (Figura 6A).

2. Preparação do enxerto (Figura 6B).

3. Incisão anteromedial oblíqua, com início a seis centímetros proximais à linha articular, dirigindo-se obliquamente até a articulação talonavicular, sobre o trajeto do tendão tibial anterior (Figura 6C).

4. Abertura dos retináculos (Figura 6D).

5. Desbridamento e preparação dos cotos tendíneos (Figura 6E).

6. Tendinoplastia com o enxerto livre unindo os cotos e tubularização (Figura 6F).

7. Fechamento dos retináculos (Figura 6G).

8. Imobilização em dorsiflexão.

Para a avaliação dos resultados foram utilizadas as escalas: graduação subjetiva de satisfação, AOFAS e Maryland.

A Tabela 1 apresenta a análise geral deste estudo.

\section{RESULTADOS}

Em relação à escala de graduação subjetiva de satisfação (presença ou ausência de dor e resultado funcional satisfatório ou insatisfatório), 12 pacientes ficaram satisfeitos e um paciente insatisfeito. Este apresentou deiscência de pele e recidiva da rotura tendo sido indicada nova cirurgia, entretanto, o paciente recusou.

O paciente 13 apresentou fraqueza muscular do tendão tibial anterior reconstruído, devido ao pouco tempo de seguimento e aderência de partes moles, porém, pela gravidade e expectativa do déficit funcional, encontravase satisfeito.

A média da escala AOFAS, no período pós-operatório, foi de 80 pontos, variando de 51 a 90 pontos.

A média da escala Maryland foi de 86 pontos, variando de 62 a 95 pontos.

A Tabela 2 relaciona o resultado obtido com o achado intraoperatório.

\section{DISCUSSÃO}

Neste trabalho retrospectivo, os 13 pacientes apresentados constituem uma pequena série de casos, porém significante perante a casuística individual dos autores. Kausch e Rutt ${ }^{(4)}$ revisaram 33 casos compilados da literatura contemporânea. A maioria dos trabalhos diz respeito a relatos de casos com a maior série de Bernstein apud Velan e Hendel ${ }^{(6)}$, citando 24 casos. Os resultados do tratamento cirúrgico nas roturas do tendão tibial anterior foram apresentados por Markarian et al ${ }^{(5)} \mathrm{em}$ uma série de 16 pacientes e, da mesma forma, Sammarco et $a l^{(7)}$ publicaram uma casuística de 19 pacientes.

A idade nesta série de pacientes portadores de tendinopatia do compartimento anterior, de diversas etiologias e dos diferentes tendões, variou de 19 a 67 anos.

Levando-se em consideração apenas o fator degenerativo, Pattern e Pun ${ }^{(8)}$ referiram, na sua revisão de literatura, a predominância da sexta e sétima décadas de pacientes com rotura do tibial anterior. Nossa amostra evidenciou $18 \%$ de pacientes que se enquadram nesta faixa. Ouzonian e Anderson ${ }^{(1)}$ e Markarian et al ${ }^{(5)}$, consideram a idade como fator decisivo na seleção pelo tratamento conservador ou cirúrgico. Nesta casuística, a opção foi pelo tratamento cirúrgico nas diferentes faixas etárias.

O gênero masculino foi o mais prevalente da mesma forma que os outros relatos ${ }^{(1,6,9,10)}$.

As tendinopatias crônicas do compartimento anterior do tornozelo (TCAT) resultam de diferentes etiologias. A etiopatogenia traumática das roturas tendíneas foi a mais encontrada e, nesta série, foi devida à laceração por ferimento cortocontuso. Rimoldi et al $^{(3)}$ relatam casos de rotura traumática por contração isotônica súbita ou ainda estiramento tendíneo por excesso de flexão plantar. As afecções de origem atraumática contempladas nesta casuística resultaram em tendinopatia crônica pelo processo degenerativo ou inflamatório de diversas etiologias: atrito, movimentos de repetição, patologia sinovial, variação anatômica (ventre muscular alongado ou acessório), etc. A pesquisa clínica de patologias sistêmicas, tais como: diabetes, hiperparatireoidismo e nefropatia, doenças reumáticas, tofo gotoso, etc., assim como o uso de corticoterapia tem sido recomendado, pois foram encontradas em outras séries comprometendo os tendões no compartimento anterior ${ }^{(5,6,10,11)}$.

O denominador comum da rotura espontânea do tendão tibial anterior, nos dois casos, foi o enfraquecimento devido ao processo degenerativo (antecedente de tendinopatia) (casos 6 e 7). 

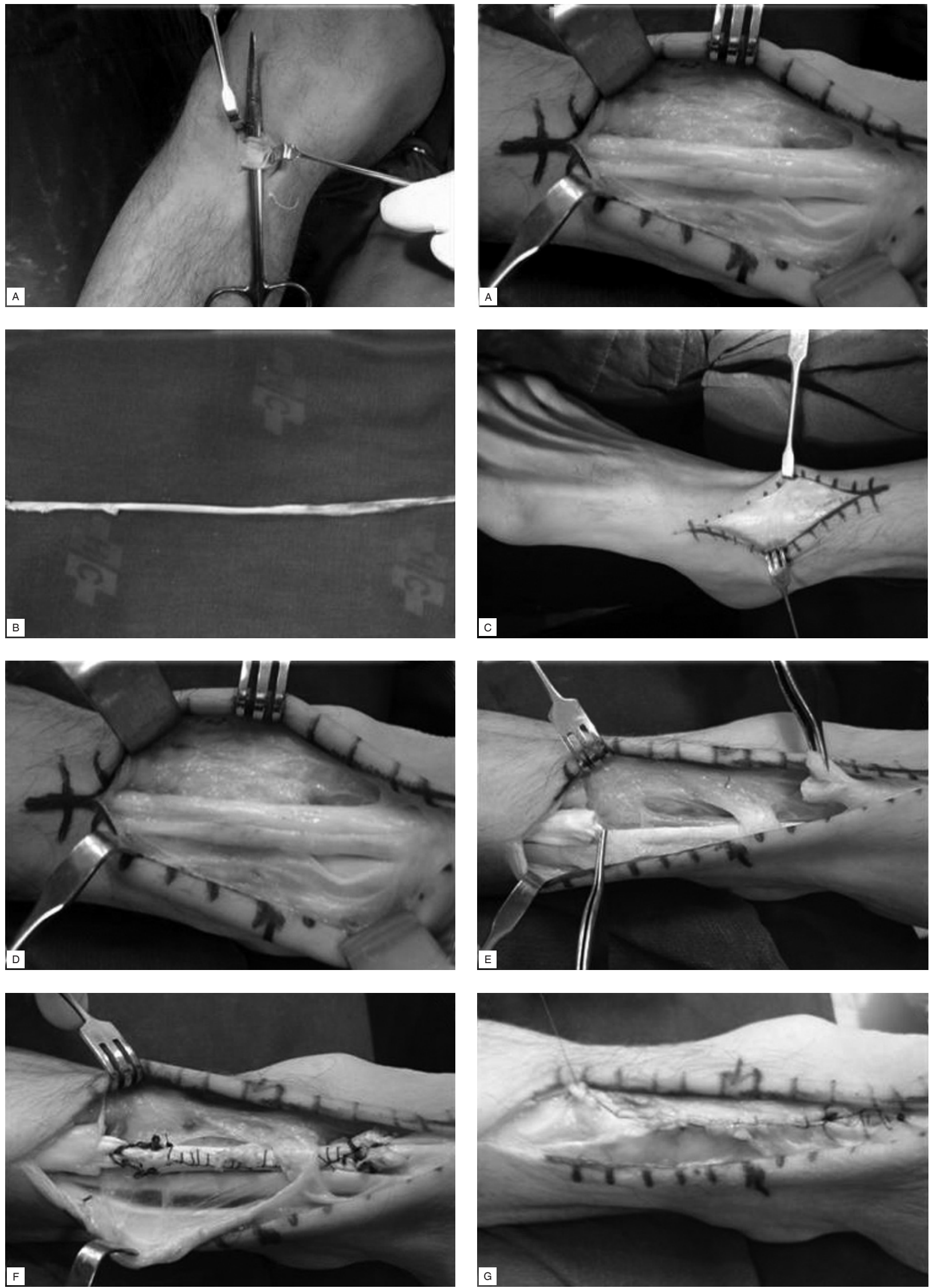

Figura 6 A - Identificação do tendão semitendíneo. B - Preparação do enxerto. C - Incisão anteromedial oblíqua. D - Abertura dos retináculos. $\mathbf{E}$ - Desbridamento e preparação dos cotos tendíneos. F - Tendinoplastia com o enxerto livre unindo os cotos e tubularização. Figura 6G - Fechamento de retináculo. 
Tabela 1 - Epidemiologia, etiopatogenia, etiologia, sinais e sintomas, antecedentes, exame físico, exame complementar e tempo decorrido do diagnóstico

\begin{tabular}{|c|c|c|c|c|c|c|c|c|c|c|}
\hline $\mathrm{N}^{0}$ & Idade & Sexo & Lado & Etiopatogenia & Etiologia & Sinais Sintomas & Antecedentes & Exame Físico & $\begin{array}{c}\text { Exame } \\
\text { Complementar }\end{array}$ & $\begin{array}{c}\text { Tempo } \\
\text { Diagnóstico }\end{array}$ \\
\hline 1 & 15 & $M$ & $\mathrm{D}$ & $T$ & FCC & CL PF-TA & & AR F IP MT & & 48 \\
\hline 2 & 28 & $\mathrm{~F}$ & $\mathrm{E}$ & NT & VA SVN & DOR IF & ATLETA CORRIDA & AS AV DOR TINEL & RM & 36 \\
\hline 3 & 22 & $\mathrm{~F}$ & D & NT & VA & AV DOR & ATLETA FUTEBOL & AV DOR TINEL & RM & 24 \\
\hline 4 & 42 & $M$ & $\mathrm{D}$ & $\mathrm{T}$ & FCC & CL PF-TA & & AR F IP MT & & 60 \\
\hline 5 & 19 & $\mathrm{~F}$ & $\mathrm{D}$ & NT & TNC & AV CL CR DOR & ATLETA GINÁSTICA & DOR IP TINEL & $\mathrm{RM}$ & 12 \\
\hline 6 & 67 & $\mathrm{M}$ & $\mathrm{D}$ & NT & DEG & CL DOR IF & & DOR IP MT & RM & 03 \\
\hline 7 & 61 & M & D & NT & DEG & CL DOR IF & & AR DOR F MT & RM & 02 \\
\hline 8 & 26 & M & D & $\mathrm{T}$ & FCC & AS CL PF-ELD $2^{\circ}, 3^{\circ}, 4^{0}, 5^{\circ}$ & IS & DOR IP TINEL & RM & 05 \\
\hline 9 & 26 & M & D & $\mathrm{T}$ & FCC & $\begin{array}{c}\text { CL PF-TA } \\
\text { ELD } \\
\text { ELH }\end{array}$ & IS & AR F IP MT & & 07 \\
\hline 10 & 47 & $M$ & $\mathrm{D}$ & $T$ & FCC & CL PF-TA & & AR F IP MT & RM & 07 \\
\hline 11 & 37 & M & $\mathrm{D}$ & $\mathrm{T}$ & FCC & AS CL PF-ELD $2^{0}, 3^{\circ}, 4^{0}, 5^{0}$ & & DOR IP TINEL & $\mathrm{RM}$ & 11 \\
\hline 12 & 19 & M & $\mathrm{D}$ & $\mathrm{T}$ & FCC & $\begin{array}{c}\text { CL PF-TA } \\
\text { ELH }\end{array}$ & & AR IP MT TINEL & RM & 01 \\
\hline 13 & 28 & M & D & $\mathrm{T}$ & FCC & $\begin{array}{l}\text { PF- TA } \\
\text { ELD } \\
\text { ELH }\end{array}$ & & AR IP PS & & 01 \\
\hline
\end{tabular}

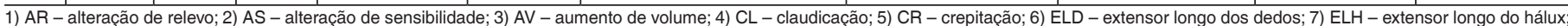

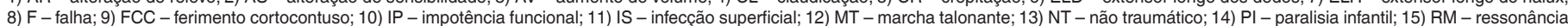

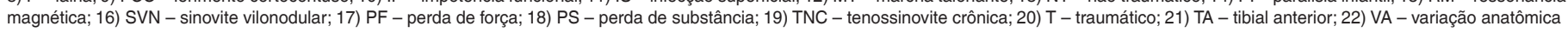

Tabela 2 - Achado operatório e resultado final

\begin{tabular}{|c|c|c|c|c|c|}
\hline $\mathrm{N}^{0}$ & Tratamento & Achado & $\begin{array}{c}\text { Seguimento } \\
\text { (meses) }\end{array}$ & $\begin{array}{c}\text { Resultado } \\
\text { objetivo }\end{array}$ & $\begin{array}{c}\text { Resultado } \\
\text { subjetivo }\end{array}$ \\
\hline 1 & SOL ELH TSJ & $\begin{array}{c}\text { AFL F 2cm } \\
\text { FBR TA }\end{array}$ & 127 & $S$ & S \\
\hline 2 & LN RVM SI & $\begin{array}{c}\text { CFP VA TA } \\
\text { ELH }\end{array}$ & 79 & S & S \\
\hline 3 & LN RVM & CFP VA TA & 67 & $S$ & $S$ \\
\hline 4 & SOL ELH & $\mathrm{F} \mathrm{4cm} \mathrm{TA}$ & 31 & $S$ & $\mathrm{~S}$ \\
\hline 5 & $\mathrm{SI}$ & ERS TN ELD & 31 & $S$ & $S$ \\
\hline 6 & SOL ELH & $\mathrm{F} \mathrm{4cm} \mathrm{TA}$ & 31 & $\mathrm{~S}$ & $S$ \\
\hline 7 & EL & $\mathrm{F} 8 \mathrm{~cm} \mathrm{TA}$ & 15 & $S$ & $S$ \\
\hline 8 & LN SOL ELH & $\begin{array}{l}\mathrm{F} 3 \mathrm{~cm} \text { ELD } \\
\text { FBR ERS }\end{array}$ & 15 & S & $S$ \\
\hline 9 & SOL ELH & $\mathrm{F} \mathrm{4cm} \mathrm{TA}$ & 13 & I & I \\
\hline 10 & EL & $\mathrm{F} 6 \mathrm{~cm}$ TA & 12 & $S$ & $S$ \\
\hline 11 & EL & F $6 \mathrm{~cm}$ ELD & 12 & $S$ & $S$ \\
\hline 12 & EL & $\mathrm{F} 7 \mathrm{~cm}$ TA & 10 & $S$ & $\mathrm{~S}$ \\
\hline 13 & EL & $\begin{array}{l}\text { F 8cm } \\
\text { TA,ELH }\end{array}$ & 04 & $S$ & S \\
\hline
\end{tabular}

1) AFL-afilamento; 2) CFP - compressão fibular profunda; 3) EL- enxerto livre; 4) ELD - extensor longo dos dedos; 5) ELH - extensor longo do hálux; 6) ERS - espessamento retináculo superior; 7) F - falha; 8) FIS - fisioterapia; 9) FBR - fibrose; 10) I-insatisfatório; 11) LN - liberação de nervo; 12) RVM - ressecção ventre muscular; 13) S - satisfatório; 14) SI - sinovectomia; 15) SOL - solidarização; 16) TA - tibial anterior; 17) TN - tenosinovite; 18) TSJ - tenossuspensão de Jones; 19) VA - variação anatômica

Os sinais clínicos que evidenciaram as roturas tendíneas foram: 1) alteração da marcha (pé caído por déficit do tendão tibial anterior e claudicação dolorosa); 2) perda da integridade à palpação dos tendões; 3) mudança do relevo do compartimento anterior; e 4) fraqueza ao teste de dorsiflexão resistida do pé ou dedos.
Quando se trata de etiopatogenia não traumática segundo Ouzoniam e Andreson ${ }^{(1)}$, o tempo decorrido entre a lesão e o diagnóstico é maior, pois estes pacientes geralmente não referiram dor e a incapacidade funcional não foi significativa devido à baixa demanda de atividade. Nos dois casos de rotura degenerativa nesta série o diagnóstico foi precoce, mas os pacientes atrasaram voluntariamente a indicação de cirurgia, tanto pela perda da função quanto pela queixa de dor.

Os casos 1 e 4 com rotura traumática eram jovens que, apesar do diagnóstico imediato, foram tratados tardiamente quando houve interesse na melhora da função.

Com relação aos antecedentes, nenhuma patologia sistêmica foi identificada. A etiopatogenia nos casos 2, 3 e 5 estava relacionada à variação anatômica (ventre muscular baixo), à presença de tumor intra-articular (sinovite vilonodular) e à atividade física intensa. A presença de sinal de Tinel foi justificada pelo efeito compressivo do aumento de conteúdo no compartimento anterior sobre o nervo fibular profundo.

A rotina do exame de imagem incluiu inicialmente a radiografia que evidenciou edema de partes moles ${ }^{(2)}$, osteófitos marginais e outras anomalias ósseas ${ }^{(3)}$. Consideramos como padrão ouro de imagem a ressonância magnética que permite o diagnóstico, estadiamento e distanciamento dos cotos. A ecografia também foi útil na impossibilidade da ressonância magnética. Aboukrat ${ }^{(12)}$ 
propôs tenoescanografia para confirmação diagnóstica das tendinopatias, tendo sido essa a única referência encontrada. A ultrassonografia, segundo Peetrons ${ }^{(13)}$, foi suficiente pela especificidade e sensibilidade.

Ouzonian e Anderson ${ }^{(1)}$ e Frigg et al ${ }^{(10)}$ utilizaram a ressonância magnética, não como método diagnóstico, mas como recurso de apuro para identificação da característica da lesão, dos detalhes do local e do distanciamento dos cotos. Este recurso de imagem foi realizado em nove pacientes e contribuiu para a escolha da via de acesso e da técnica cirúrgica, assim como no diagnóstico das variações anatômicas.

Nos pacientes ativos de etiologia traumática ou degenerativa, com rotura total, há uma tendência na literatura de que o tratamento cirúrgico seja de eleição.

Quando o diagnóstico da rotura é precoce, pode-se realizar anastomose término-terminal dos cotos. Nas lesões crônicas, quando não é possível a sutura direta, poder-se-ia realizar o deslizamento em "V-Y" ou a solidarização ou o enxerto livre. O deslizamento em "V-Y" não foi indicado nos tendões do compartimento anterior pelo pequeno diâmetro, o que resultaria em dificuldade técnica. A solidarização passou a ser um recurso factível e eficaz tendo sido praticada com os tendões adjacentes, tais como ELH, ELD e TA, quando o distanciamento dos cotos não excedia $4 \mathrm{~cm}$.

Os enxertos poderiam ser autógenos ou autólogos. Nesta série foi utilizado, apenas, o enxerto autógeno, pois não dispomos de banco de tecidos, mas esta poderá ser uma opção no futuro. O enxerto livre foi utilizado quando a distância maior que $5 \mathrm{~cm}$ entre os cotos tornava impraticável a sutura término-terminal ou a solidarização.

A escolha pelo enxerto livre do tendão do semitendíneo foi devida às suas características similares de espessura e resistência, além de um tamanho conveniente e fácil aquisição. O emprego do tendão semitendíneo tem sido recurso consagrado nas reconstruções ligamentares do joelho, pela pequena morbidade e pouca interferência na

\section{REFERÊNCIAS}

1. Ouzonian TJ, Anderson R. Anterior tibial tendon rupture. Foot Ankle Int. 1995;16(7): 406-10.

2. Kashyap S, Prince R. Spontaneous rupture of the tibialis anterior tendon: a case report. Clin Orthop Relat Res. 1987;(216):159-61.

3. Rimoldi RL, Oberlander MA, Waldrop JI, Hunter SC. Acute rupture of the tibialis anterior tendon: a case report. Foot Ankle Int. 1991;12(3):176-7.

4. Kaush T, Rutt J. Subcutaneous rupture of the tibialis anterior tendon: review of the literature and a case report. Arch Orthop Trauma Surg. 1998;117(4-5):290-3.

5. Markarian GG, Kelikian AS, Brage M, Trainor T, Dias L. Anterior tibialis tendon ruptures: an outcome analysis of operative versus nonoperative treatment. Foot Ankle Int. 1998;19(12):792-802.

6. Velan GJ, Hendel D. Degenerative tear of the tibialis anterior tendon after corticosteroid injection - augmentation with the extensor hallucis longus tendon, case report. Acta Orthop Scand. 1997;68(3):308-9. força flexora. O princípio de não desequilibrar a ação da musculatura extrínseca do pé e tornozelo ficou satisfeito, pois não utilizamos transferências que alterariam esta dinâmica. A sutura do enxerto livre aos cotos tendíneos se fez na máxima tensão possível e a imobilização foi colocada em dorsiflexão do tornozelo a $95^{\circ}$ em $30^{\circ}$ dos dedos. A integração do enxerto livre ocorreu em todos os casos.

O sítio de rotura mais comumente encontrado por Pattern e Pun ${ }^{(8)}$ está de 0,5 a 3cm proximal à inserção do tendão tibial anterior. A média encontrada neste estudo foi de $5 \mathrm{~cm}$ variando de 2 a $8 \mathrm{~cm}$ deste ponto de referência.

O tempo decorrido do diagnóstico ao tratamento, independentemente do fator ser traumático ou degenerativo, foi tardio (média de 19 meses) devido a diferentes motivos. A baixa demanda funcional, a dor de pequena intensidade e a adaptação à marcha com o pé caído (compensada pelo uso de órtese) podem ser justificativas para a procura tardia.

Os resultados desta série indicam que o tratamento cirúrgico foi eficiente no restabelecimento da função e no alívio da dor. O retorno às atividades pré-lesionais foi obtida em $92 \%$ dos casos.

Nas tendinopatias do compartimento anterior, quer de origem traumática ou não, o tratamento cirúrgico, também, mostrou-se a opção necessária quando se pretendia o restabelecimento funcional.

\section{CONCLUSÃO}

1 - O tratamento cirúrgico é eficaz para recuperação funcional.

2 - Diversas técnicas devem ser praticadas para atender o espectro de gravidade das roturas tendíneas e tratar os diagnósticos diferencias possíveis da TCAT.

3 - A opção do enxerto livre do tendão semitendíneo é eficiente nas falhas maiores que cinco centímetros e da mesma forma a solidarização nas distâncias menores entre os cotos.

7. Sammarco VJ, Sammarco GJ, Henning C, Chaim S. Surgical repair of acute and chronic tibialis anterior tendon ruptures. J Bone Joint Surg Am. 2009;91(2):325-32.

8. Pattern A, Pun WK. Spontaneous rupture of the tibialis anterior tendon: a case report and literature review. Foot Ankle Int. 2000;21(8):697-9.

9. Goehring $\mathrm{M}$, Liakos $\mathrm{P}$. Long-term outcomes following anterior tibialis tendon reconstruction with hamstring autograft in a series of 3 cases. J Foot Ankle Surg. 2009;48(2):196-202.

10. Frigg AM, Valderrabano V, Kundert HP, Hintermann B. Combined anterior tibial tendon rupture and posterior tibial tendon dysfunction in advanced flatfoot. J Foot Ankle Surg. 2006;45(6):431-5.

11. Dooley BJ, Kudelka P, Menelaus MB. Subcutaneous rupture of the tendon of tibialis anterior. J Bone Joint Surg Am. 1980; 62(4):471-2.

12. Aboukrat P. Tendonopathies du pied. Rev Prat. 1997;47(1):56-61.

13. Peetrons P. Lesions du tendon tibial anterieur en echographie: rapport de deux cas. JBR-BTR. 1999;82(4):157-8. 